\title{
Strategies for Early Non-response to Antipsychotic Drugs in the Treatment of Acute-phase Schizophrenia
}

\author{
Kotaro Hatta ${ }^{1}$, Hiroto Ito $^{2}$ \\ 'Department of Psychiatry, Juntendo University Nerima Hospital, Tokyo, ${ }^{2}$ Department of Social Psychiatry, National Center of Neurology \\ and Psychiatry, Tokyo, Japan
}

\begin{abstract}
As a strategy for antipsychotic treatment of schizophrenia, monotherapy is clearly optimal when both effective and tolerated. When a patient fails to respond to an adequate dose of an antipsychotic, alternatives include switching, administering a higher dose (above the licensed dose), polypharmacy or clozapine. Clozapine is the only option with established efficacy, but is less manageable than other antipsychotics. We therefore reviewed other options, focusing on the treatment of acute-phase schizophrenia. According to recent evidence, an antipsychotic may be viewed as ineffective within 1-4 weeks in acute-phase practice, although some differences may exist among antipsychotics. Whether a switching strategy is effective might depend on the initial antipsychotic and which antipsychotic is switched to. As weak evidence points toward augmentation being superior to continuation of the initial antipsychotic, inclusion of augmentation arms in larger studies comparing strategies for early non-responders in the acute-phase is justified. With respect to high-doses, little evidence is available regarding acute-phase treatment, and the issue remains controversial. Although evidence for antipsychotic switching, augmentation, and high-doses has gradually been accumulating, more studies performed in real clinical practice with minimal bias are required to establish strategies for early non-response to an antipsychotic drug in the treatment of acute-phase schizophrenia.
\end{abstract}

KEY WORDS: Drug switching; Augmentation; High-dose; Polypharmacy.

\section{INTRODUCTION}

As a strategy for antipsychotic treatment of schizophrenia, monotherapy is clearly optimal when both effective and tolerated. When a patient fails to respond to an adequate dose of an antipsychotic, however, the alternatives include switching, administering a dose higher than the licensed dose, polypharmacy or clozapine. Clozapine is the only option with established efficacy, but is less manageable than other antipsychotics, with a relatively high frequency of clozapine-induced agranulocytosis. Other options therefore need to be comprehensively evaluated.

Little evidence has been accumulated from acute-phase practice, as enrolling acute psychotic and agitated patients in randomized clinical trials is challenging, particularly when using a double-blind design. However, most clinical guidelines are based on findings from double-blind trials,

\footnotetext{
Received: December 27, 2013 / Revised: February 4, 2014

Accepted: February 6, 2014

Address for correspondence: Kotaro Hatta, MD, PhD

Department of Psychiatry, Juntendo University Nerima Hospital,

Takanodai 3-1-10, Nerima-ku, Tokyo 177-8521, Japan

Tel: +81-3-5923-3111, Fax: +81-3-5923-3217

E-mail: khatta@juntendo.ac.jp
}

which include ideal patients. ${ }^{1)}$ Such guidelines are therefore not necessarily useful in actual clinical practice. From this practical perspective, strategies for early non-response to an antipsychotic drug in acute-phase schizophrenia are discussed (Fig. 1).

\section{MAIN DISCOURSE}

\section{How Long Should an Antipsychotic Be Trialed before Being Viewed as Ineffective in the Treatment of Acute-phase Schizophrenia?}

How long an antipsychotic should be trialed before being viewed as ineffective is a key unanswered question in clinical trials for patients with schizophrenia. ${ }^{2)}$ Although previous studies have identified early non-response as a robust predictor of subsequent non-response with continued treatment of the same medication, ${ }^{3-7)}$ those studies were retrospective in nature. The first prospective study was performed by Kinon et $a l^{8)}$ finding early response/non-response to risperidone at 2 weeks as a reliable clinical marker of subsequent clinical outcomes (Table 1).

(c) This is an Open-Access article distributed under the terms of the Creative Commons Attribution Non-Commercial License (http://creativecommons.org/licenses/by-nc/3.0) which permits unrestricted non-commercial use, distribution, and reproduction in any medium, provided the original work is properly cited. 
We examined whether early response/non-response to risperidone according to the Clinical Global ImpressionsImprovement Scale (CGI-I) at 2 weeks could predict subsequent response in the treatment of acute-phase schizophrenia. ${ }^{9)}$ At 4 weeks, $81 \%$ of risperidone early responders achieved $\geq 50 \%$ response, whereas only $9 \%$ of early non-responders staying on risperidone achieved $\geq 50 \%$ response, with a negative likelihood ratio of 0.057 . In contrast, the negative likelihood ratio for the prediction of $\geq 50 \%$ response at 4 weeks based on early response status to olanzapine at 2 weeks was 0.28 , suggesting this prediction was not necessarily applicable to olanzapine.

Levine and Leucht ${ }^{10)}$ recently reanalyzed data from a double-blind, randomized, multicenter, international clinical trial comparing the effectiveness of treatment with olanzapine and haloperidol in recent-onset psychotic patients, in which patients were followed up to 84 weeks. ${ }^{11)}$ They reported that early response (i.e., within 2 weeks) is marked by up to 39 weeks of longer subsequent symptom response than non-response (i.e., in the initial 4 weeks), and infrequently differs to delayed-response (i.e., 3-4 weeks). As half of patients were allocated to receive olanzapine, results from the reanalysis may not necessarily be applicable to risperidone. Furthermore, participants were defined as patients with recent-onset psychosis with an interval since first onset of psychotic symptoms of $\geq 1$ month but $\leq 60$ months prior to study entry, indicating that patients were not necessarily in the acute phase. Mean total scores for the positive and negative syndrome scale (PANSS) at baseline were relatively low, i.e.: early-responder, 57.24; delayed-responder, 61.93; and non-responder, 78.32, also indicating that most cases were not in the acute phase.

Derks et al. ${ }^{12)}$ reported data from 299 first-episode patients who completed the full 12-month European First-Episode Schizophrenia Trial, finding that remission status was correctly predicted in $61 \%$ of patients based on baseline and 2-week assessments, and this percentage increased to $63 \%$ and $68 \%$, respectively, with the inclusion of 4- and 6-week assessments, respectively. Accordingly, they concluded that earlier 2-week measures of response are associated with remission, and that as the increase in prediction accuracy by including 4- and 6-week assessments is modest, whether such improvement is clinically relevant remains uncertain.

Focusing on acutely psychotic patients, O'Gorman et $a l .{ }^{13)}$ reported that from two separate pooled analyses of two placebo-controlled and two active comparator (risperidone and olanzapine) randomized trials of ziprasi- done in schizophrenia, non-improvement in weeks 1 or 2 was highly predictive of non-response, and week 2 improvement was more reliably predictive of subsequent outcome than week 1 improvement, with high sensitivity and specificity. Giegling et al. ${ }^{14)}$ reported that a PANSS reduction $\leq 16 \%$ at 1 week predicts non-response at 3 weeks of treatment with haloperidol (specificity 92\%, sensitivity $82 \%$ ), and, conversely, a PANSS reduction $\geq 23 \%$ at 1 week of treatment predicts response at 3 weeks, with $84 \%$ specificity and $86 \%$ sensitivity. Considering our results that non-response to risperidone at 2 weeks can predict subsequent response in newly admitted patients with acute schizophrenia, and that significant response to olanzapine does not seem to occur until 4 weeks, ${ }^{9)}$ the duration for which an antipsychotic should be trialed before being viewed as ineffective might depend on the kind of antipsychotic, such as affinity and specificity for dopamine D2 receptors. According to these findings, an antipsychotic may be able to be viewed as ineffective within 1-4 weeks in acute-phase practice, although some differences may exist among specific antipsychotics.

\section{Is Switching from an Antipsychotic to Another More Effective Than Staying on the Initial Antipsychotic in Early Non-responders?}

Before we presume that identifying early non-responders minimizes prolonged exposure to suboptimal or ineffective treatment strategies, alternative treatment strategies such as "switching", "augmentation", and "highdose" antipsychotics should be evaluated. First, we reviewed whether patients who are early non-responders to an antipsychotic and are then switched to another antipsychotic would show significantly greater improvement in psychopathology, compared with those staying on the initial antipsychotic.

In a switching study of first-episode patients with schizophrenia who showed residual symptoms following treatment with risperidone, Takahashi et al. ${ }^{15)}$ reported the rate of responders to olanzapine was $29.3 \%$. They also reported that in another switching study of first-episode patients with schizophrenia who experienced residual symptoms following treatment with olanzapine, the rate of response to risperidone was $35.3 \% .{ }^{16)}$ However, the patients enrolled were those who were able to complete 12 weeks of treatment with the initial antipsychotic, indicating that they were not in the acute phase. Furthermore, those studies lacked a control group of patients staying on the initial antipsychotic, and we therefore cannot conclude that 
A 16 Citations identified through database searching

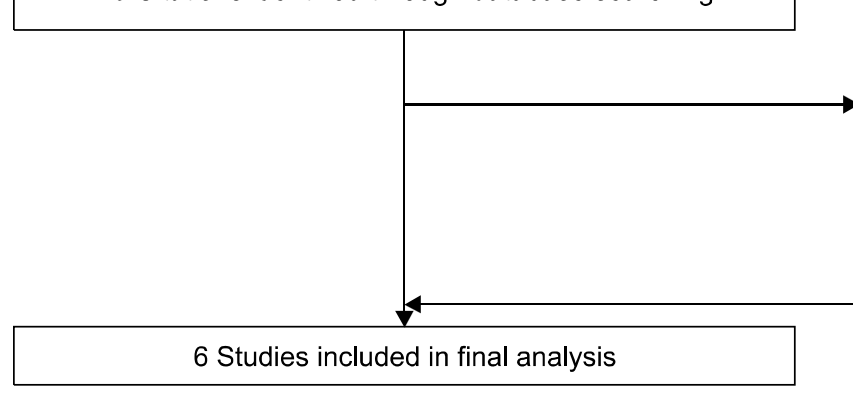

13 Articles excluded with reasons: Retrospective nature $(n=4)$ Appropriate outcome not reported $(n=4)$

It was not associated with early response $(n=2)$

Investigation of long-acting injection $(n=1)$

Investigation of depressive symptoms $(n=1)$ Duplicative $(n=1)$

3 Articles included after hand search

B

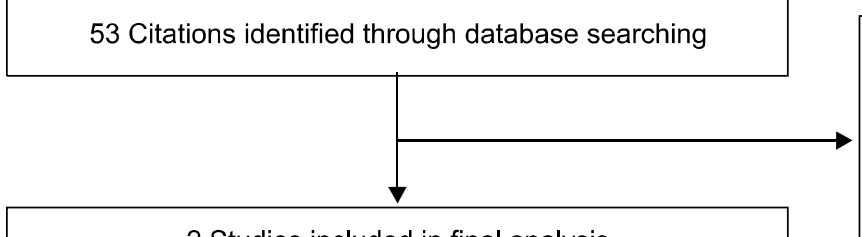

2 Studies included in final analysis
51 Articles excluded with reasons:

It was not associated with early non-response $(n=47)$

Duplicative $(n=1)$

Retrospective nature $(n=1)$

It was not associated with acute-phase $(n=1)$

Appropriate outcome not reported $(n=1)$
C

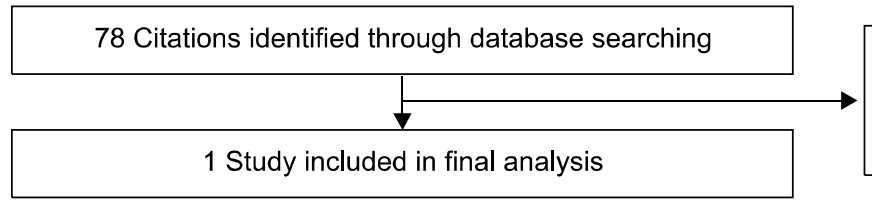

77 Articles excluded with reasons:

It was not associated with early non-response $(n=76)$

Investigation of antidepressant augmentation $(n=1)$

D

32 Citations identified through database searching

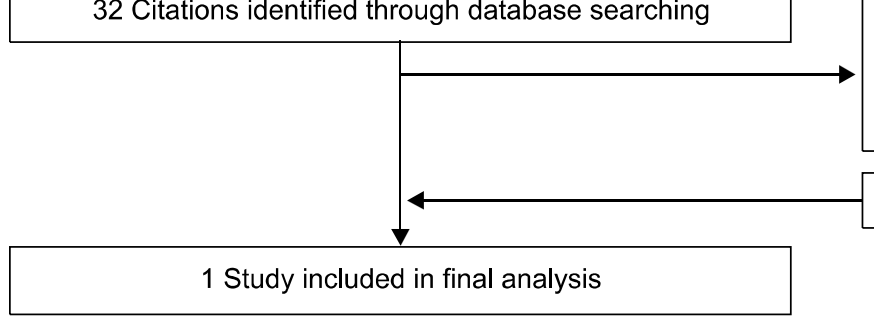

32 Articles excluded with reasons:

It was not associated with acute-phase $(n=29)$

It was not associated with early non-response $(n=2)$

Investigation of depressive symptoms $(n=1)$

1 Article included after hand search

Fig. 1. Flowchart of the systematic literature search. We conducted a computerized literature search in the PubMed/Medline up to December 14, 2013. (A) Clinical trials of early response to antipsychotics in schizophrenia. The search terms were "antipsychotic", "schizophrenia", "clinical trial", and "early response". (B) Randomized clinical trials of antipsychotic switching in early non-responders in schizophrenia. The search terms were "antipsychotic", "schizophrenia", "randomized clinical trial", and "switch". (C) Randomized clinical trials of antipsychotic augmentation in early non-responders in schizophrenia. The search terms were "antipsychotic", "schizophrenia", "randomized clinical trial", and "augmentation". (D) Randomized clinical trials of high-dose antipsychotics in early non-responders in schizophrenia. The search terms were "antipsychotic", "schizophrenia", "randomized clinical trial", and "high-dose".

switching is indeed a beneficial option.

Although Essock et al. ${ }^{17)}$ and Rosenheck et al. ${ }^{18)}$ reported no benefit of switching antipsychotic medications in analyses of the Clinical Antipsychotic Trials of Intervention Effectiveness (CATIE) trial, which included a control group, those analyses were post hoc exploratory nature.

The first randomized, double-blind study of whether 'switching' early non-responders to another antipsychotic represents a better strategy than 'staying' was reported by
Kinon et al. ${ }^{8)}$ They showed that switching to risperidone in early non-responders to olanzapine at week 2 resulted in a small but significantly greater reduction in PANSS total score and in depressive symptoms. The significant difference in PANSS total score at end point mean change at week 12 was reportedly 3.49 . We failed to show any robust advantage of the switching strategy for early non-responders within the ordinary doses of risperidone or olanzapine in acute-phase clinical practice, probably due to the lack of statistical power for the randomized phase of the 


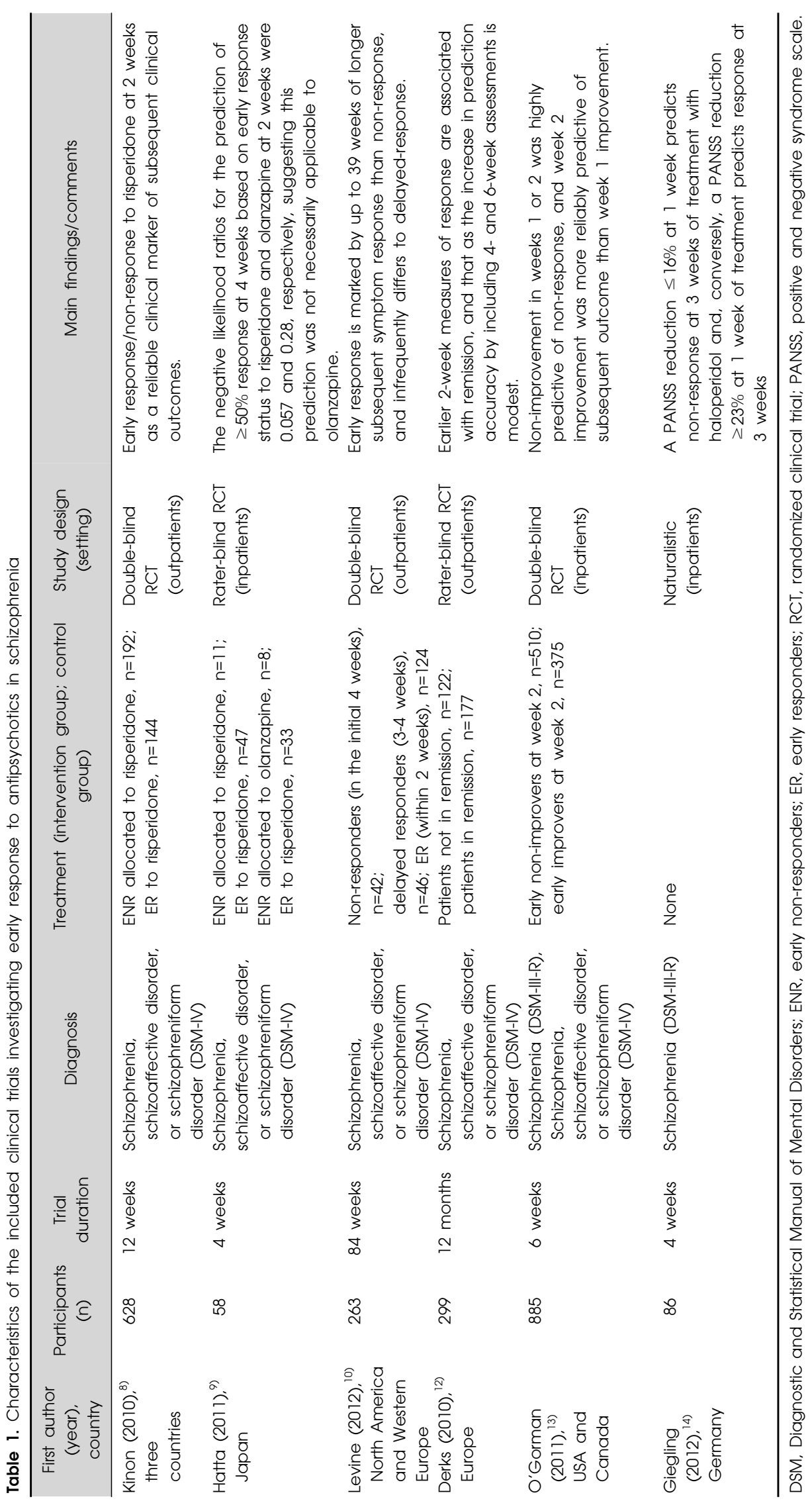


study. ${ }^{9)}$

Agid et al. ${ }^{19)}$ reported that from data gathered from a treatment algorithm implemented in patients with first-episode schizophrenia that employs two antipsychotic trials at increasing doses before clozapine, the percentage response for subjects who switched from olanzapine to risperidone was $4.0 \%$, compared to $25.7 \%$ for those who switched from risperidone to olanzapine. Thus, whether a switching strategy is effective might depend on the initial antipsychotic administered and the antipsychotic switched to.

\section{Is Add-on Therapy with Another Antipsychotic More Effective than Staying on the Initial Antipsychotic Monotherapy in Early Non-responders to the Initial Antipsychotic?}

A substantial proportion of schizophrenia patients receive more than one antipsychotic. ${ }^{20-22)}$ The current problem is that the degree of polypharmacy being practiced seems far in excess of the supporting data. ${ }^{2)}$ In a systematic review of 19 randomized studies, the pooled odds ratio suggested a small effect favoring combination treatment, and positive effects appear to have been associated with studies using clozapine combinations. ${ }^{23)}$ However, clozapine is not tolerated by some patients. Studies combining non-clozapine second-generation antipsychotics with each other and with the first-generation antipsychotics utilized most in clinical practice are thus required. ${ }^{23)}$

Kotler et $a .^{24)}$ indicated no significant differences in changes to positive or negative symptomatology between patients receiving a combined regimen of olanzapine with sulpiride augmentation and those receiving olanzapine monotherapy among chronic schizophrenia patients unresponsive to olanzapine. Kane et $\mathrm{al}^{25)}$ reported that addition of aripiprazole to either risperidone or quetiapine in 323 patients showed no greater efficacy than placebo added to either risperidone or quetiapine. In contrast, Essock et $a l^{26)}$ reported that patients assigned to a switch to monotherapy displayed shorter times to all-cause treatment discontinuation than those assigned to remain on polypharmacy. These studies were indicators of what could happen with antipsychotic combinations in chronic-phase patients.

We have presented the first randomized clinical trial of olanzapine augmentation of risperidone in patients non-responsive to risperidone monotherapy in the acute phase. ${ }^{27)}$ In the study, early response was defined as CGI-I $\leq 3$ following 2 weeks of treatment, and early non-responders were then allocated to receive either augmenta- tion with olanzapine (RIS+OLZ group) or increased risperidone dose (RIS+RIS group). Although time to treatment discontinuation for any cause was significantly shorter in the RIS+RIS group than in early responders to risperidone, no significant difference was evident between the RIS+OLZ group and early responders to risperidone. These outcomes justify the inclusion of augmentation arms in additional, larger studies comparing strategies for early non-responders in the treatment of acute-phase schizophrenia.

\section{Are High-dose Antipsychotics Effective in Patients Who Do Not Achieve Sufficient Response to Conventional Doses in the Treatment of Acute-phase Schizo- phrenia?}

In clinical practice, nearly $50 \%$ of olanzapine prescriptions in the United States was reportedly above 20 $\mathrm{mg} / \mathrm{day}^{28)}$ and the median recommended dose for olanzapine by U.S. experts was $30 \mathrm{mg} /$ day. ${ }^{29)}$ The upper limit of olanzapine dose in the CATIE study, in which olanzapine was the most effective in terms of rates of discontinuation, was designed to be $30 \mathrm{mg} /$ day. $^{30)} \mathrm{In}$ chronic schizophrenia inpatients showing suboptimal response to treatment, one randomized controlled trial allowed the use of high doses of olanzapine. ${ }^{31)}$ In that study, clozapine and olanzapine were superior to haloperidol, but no obvious superiority of risperidone over haloperidol was seen. In patients with treatment-resistant schizophrenia, olanzapine at higher-than-customary doses reportedly demonstrated similar efficacy to clozapine ${ }^{32)}$ or was less effective than clozapine. ${ }^{33)}$

For acute-phase patients, we presented the first randomized clinical trial that examined whether olanzapine at $\leq 40 \mathrm{mg} /$ day would be superior to risperidone at $\leq 12$ $\mathrm{mg} /$ day. ${ }^{34)}$ As the numbers of patients allocated to each treatment group did not reach the required numbers set by power analysis, the results were not conclusive. Although time to treatment discontinuation due to any cause did not differ between treatment groups, more than half of cases that were non-responsive to conventional doses showed moderate improvement on subsequent treatment with high doses. In the study, serum olanzapine concentrations at the time of oral $20 \mathrm{mg} /$ day could be obtained from 5 out of 7 patients who subsequently required high-dose olanzapine. All values were $>30 \mathrm{ng} / \mathrm{mL}$, which were appropriate with regard to a therapeutic range of $20-50 \mathrm{ng} / \mathrm{mL}$. The reason for requiring high-dose olanzapine in the treatment of acute-phase schizophrenia thus cannot be explained simply based on pharmacokinetics. Furthermore, 
2 of 5 patients who subsequently required high-dose olanzapine, and from whom serum olanzapine concentrations could be obtained, were antipsychotic-naïve, suggesting that the etiology of dopamine supersensitivity psychosis was not applicable in those cases. ${ }^{35)}$

\section{CONCLUSION}

To establish strategies for early non-response to an antipsychotic drug in the treatment of acute-phase schizophrenia, evidence for antipsychotic switching, augmentation, or high-doses has gradually been accumulating. More studies performed in actual clinical practice with minimal bias are required to assist clinicians in making rational treatment decisions.

\section{Acknowledgments}

This work was supported by grants from the Ministry of Health, Welfare, and Labor of the Japanese Government (Comprehensive Research on Disability Health and Welfare, H23-008). The sponsor of the study had no role in study design, data collection, data analysis, data interpretation, writing of the report, and the decision to submit the paper for publication.

Dr. Hatta has received lecture honoraria for Eli Lilly, Janssen, Dainippon-Sumitomo, Otsuka, Astellas, TanabeMitsubishi, and Meiji, and served as consultant for Eli Lilly and Janssen. Dr. Ito declares no potential conflict of interest.

\section{REFERENCES}

1. Zarin DA, Young JL, West JC. Challenges to evidence-based medicine: a comparison of patients and treatments in randomized controlled trials with patients and treatments in a practice research network. Soc Psychiatry Psychiatr Epidemiol 2005;40:27-35.

2. Kane JM, Leucht S. Unanswered questions in schizophrenia clinical trials. Schizophr Bull 2008;34:302-309.

3. Ascher-Svanum H, Nyhuis AW, Faries DE, Kinon BJ, Baker RW, Shekhar A. Clinical, functional, and economic ramifications of early nonresponse to antipsychotics in the naturalistic treatment of schizophrenia. Schizophr Bull 2008;34:1163-1171.

4. Correll CU, Malhotra AK, Kaushik S, McMeniman M, Kane JM. Early prediction of antipsychotic response in schizophrenia. Am J Psychiatry 2003;160:2063-2065.

5. Kinon BJ, Chen L, Ascher-Svanum H, Stauffer VL, Kollack-Walker S, Sniadecki JL, et al. Predicting response to atypical antipsychotics based on early response in the treatment of schizophrenia. Schizophr Res 2008;102:230 240.

6. Leucht S, Busch R, Kissling W, Kane JM. Early prediction of antipsychotic nonresponse among patients with schizo- phrenia. J Clin Psychiatry 2007;68:352-360.

7. Leucht S, Shamsi SA, Busch R, Kissling W, Kane JM. Predicting antipsychotic drug response - replication and extension to six weeks in an international olanzapine study. Schizophr Res 2008;101:312-319.

8. Kinon BJ, Chen L, Ascher-Svanum H, Stauffer VL, Kollack-Walker S, Zhou W, et al. Early response to antipsychotic drug therapy as a clinical marker of subsequent response in the treatment of schizophrenia. Neuropsychopharmacology 2010;35:581-590.

9. Hatta K, Otachi T, Sudo Y, Hayakawa T, Ashizawa Y, Takebayashi $\mathrm{H}$, et al; JAST Study Group. Difference in early prediction of antipsychotic non-response between risperidone and olanzapine in the treatment of acute-phase schizophrenia. Schizophr Res 2011;128:127-135.

10. Levine SZ, Leucht S. Early symptom response to antipsychotic medication as a marker of subsequent symptom change: an eighteen-month follow-up study of recent episode schizophrenia. Schizophr Res 2012;141:168-172.

11. Lieberman JA, Tollefson G, Tohen M, Green AI, Gur RE, Kahn R, et al; HGDH Study Group. Comparative efficacy and safety of atypical and conventional antipsychotic drugs in first-episode psychosis: a randomized, double-blind trial of olanzapine versus haloperidol. Am J Psychiatry 2003; 160:1396-1404.

12. Derks EM, Fleischhacker WW, Boter H, Peuskens J, Kahn RS; EUFEST Study Group. Antipsychotic drug treatment in first-episode psychosis: should patients be switched to a different antipsychotic drug after 2, 4, or 6 weeks of nonresponse? J Clin Psychopharmacol 2010;30:176-180.

13. O'Gorman C, Kapur S, Kolluri S, Kane J. Early improvement on antipsychotic treatment as a predictor of subsequent response in schizophrenia: analyses from ziprasidone clinical studies. Hum Psychopharmacol 2011;26:282-290.

14. Giegling I, Porcelli S, Balzarro B, Andrisano C, Schäfer M, Möller HJ, et al. Antipsychotic response in the first week predicts later efficacy. Neuropsychobiology 2012;66:100105.

15. Takahashi H, Kamata M, Yoshida K, Ishigooka J, Higuchi H. Switching to olanzapine after unsuccessful treatment with risperidone during the first episode of schizophrenia: an open-label trial. J Clin Psychiatry 2006;67:1577-1582.

16. Takahashi H, Yoshida K, Ishigooka J, Higuchi H. Switching to risperidone after unsuccessful treatment of olanzapine in the first-episode schizophrenia: an open trial. Prog Neuropsychopharmacol Biol Psychiatry 2006;30:1067-1072.

17. Essock SM, Covell NH, Davis SM, Stroup TS, Rosenheck RA, Lieberman JA. Effectiveness of switching antipsychotic medications. Am J Psychiatry 2006;163:2090-2095.

18. Rosenheck RA, Davis S, Covell N, Essock S, Swartz M, Stroup S, et al. Does switching to a new antipsychotic improve outcomes? Data from the CATIE Trial. Schizophr Res 2009;107:22-29.

19. Agid O, Schulze L, Arenovich T, Sajeev G, McDonald K, Foussias G, et al. Antipsychotic response in first-episode schizophrenia: efficacy of high doses and switching. Eur Neuropsychopharmacol 2013;23:1017-1022.

20. Edlinger M, Hausmann A, Kemmler G, Kurz M, Kurzthaler I, Walch T, et al. Trends in the pharmacological treatment of patients with schizophrenia over a 12 year observation period. Schizophr Res 2005;77:25-34.

21. Correll CU. Antipsychotic polypharmacy, Part 2: Why use 2 antipsychotics when 1 is not good enough? J Clin Psychiatry 2008;69:860-861.

22. Choi HJ, Jung SH, Kang MH, Lee JS, Bae JN, Kim CE. 
Antipsychotics prescribing patterns of patients with schizophrenia admitted to Korean general hospital psychiatric unit: 2001 to 2008. Clin Psychopharmacol Neurosci 2011;9: $17-22$.

23. Correll CU, Rummel-Kluge C, Corves C, Kane JM, Leucht S. Antipsychotic combinations vs monotherapy in schizophrenia: a meta-analysis of randomized controlled trials. Schizophr Bull 2009;35:443-457.

24. Kotler M, Strous RD, Reznik I, Shwartz S, Weizman A, Spivak B. Sulpiride augmentation of olanzapine in the management of treatment-resistant chronic schizophrenia: evidence for improvement of mood symptomatology. Int Clin Psychopharmacol 2004;19:23-26.

25. Kane JM, Correll CU, Goff DC, Kirkpatrick B, Marder SR, Vester-Blokland E, et al. A multicenter, randomized, doubleblind, placebo-controlled, 16-week study of adjunctive aripiprazole for schizophrenia or schizoaffective disorder inadequately treated with quetiapine or risperidone monotherapy. J Clin Psychiatry 2009;70:1348-1357.

26. Essock SM, Schooler NR, Stroup TS, McEvoy JP, Rojas I, Jackson C, et al; Schizophrenia Trials Network. Effectiveness of switching from antipsychotic polypharmacy to monotherapy. Am J Psychiatry 2011;168:702-708.

27. Hatta K, Otachi T, Sudo Y, Kuga H, Takebayashi H, Hayashi H, et al; JAST Study Group. A comparison between augmentation with olanzapine and increased risperidone dose in acute schizophrenia patients showing early nonresponse to risperidone. Psychiatry Res 2012;198:194-201.

28. Citrome L, Jaffe A, Levine J. Datapoints: The ups and downs of dosing second-generation antipsychotics. Psychiatr Serv 2007;58:11.

29. Gardner DM, Murphy AL, O'Donnell H, Centorrino F,
Baldessarini RJ. International consensus study of antipsychotic dosing. Am J Psychiatry 2010;167:686-693.

30. Lieberman JA, Stroup TS, McEvoy JP, Swartz MS, Rosenheck RA, Perkins DO, et al; Clinical Antipsychotic Trials of Intervention Effectiveness (CATIE) Investigators. Effectiveness of antipsychotic drugs in patients with chronic schizophrenia. N Engl J Med 2005;353:1209-1223.

31. Volavka J, Czobor P, Sheitman B, Lindenmayer JP, Citrome $\mathrm{L}$, McEvoy JP, et al. Clozapine, olanzapine, risperidone, and haloperidol in the treatment of patients with chronic schizophrenia and schizoaffective disorder. Am J Psychiatry 2002;159:255-262.

32. Meltzer HY, Bobo WV, Roy A, Jayathilake K, Chen Y, Ertugrul A, et al. A randomized, double-blind comparison of clozapine and high-dose olanzapine in treatment-resistant patients with schizophrenia. J Clin Psychiatry 2008;69:274285.

33. Kumra S, Kranzler H, Gerbino-Rosen G, Kester HM, De Thomas C, Kafantaris V, et al. Clozapine and "high-dose" olanzapine in refractory early-onset schizophrenia: a 12-week randomized and double-blind comparison. Biol Psychiatry 2008;63:524-529.

34. Hatta K, Takebayashi H, Sudo Y, Katayama S, Kasuya M, Shirai Y, et al; JAST Study Group. The possibility that requiring high-dose olanzapine cannot be explained by pharmacokinetics in the treatment of acute-phase schizophrenia. Psychiatry Res 2013;210:396-401.

35. Iyo M, Tadokoro S, Kanahara N, Hashimoto T, Niitsu T, Watanabe $\mathrm{H}$, et al. Optimal extent of dopamine D2 receptor occupancy by antipsychotics for treatment of dopamine supersensitivity psychosis and late-onset psychosis. J Clin Psychopharmacol 2013;33:398-404. 